\section{ON THE RESULTS OF THYROTOMY FOR THE REMOVAL OF GROWTHS FROM THE LARYNX.}

BY MORELL MACKENZIE, M.D.Lond.,

Physician to the Hospital for Diseases of the Throat ; Senior Assistant-Physician to the London Hospital ; etc.

PREFACE.

THE current volume of the Medico-Chirurgical Transactions contains a paper by Mr. Arthur Durham, "On Section of the Laryngeal Cartilages for the Removal of Morbid Growths." The greater part of the article consists of a translation of certain portions of Planchon's Faits Cliniques de Laryngotomic, but an elaborate attempt is also made to show that my essay on Growths in the Larynx (Churchill, I871) contains numerous inaccuracies.

Not being a Fellow of the Royal Medical and Chirurgical Society, I endeavoured to reply to the charges at the Clinical Society; but, the members of that body regarding my contribution as controversial rather than clinical, and as an answer to an attack made at another society, it was decided to withdraw the paper. With Mr. Durham's co-operation, I then offered the article, modified to some extent both as regards matter and manner, to the Royal Medical and Chirurgical Society. The Council submitted it to a referee, who proposed to omit or seriously modify numerous passages-in fact, nearly all those matters in dispute between Mr. Durham and myself. With a view of giving the same publicity to the defence as had already been given to the attack, I should have been willing to make some alteration; but $I$ found that the changes proposed by the referee were of so fundamental a character, that my paper would have been deprived of its most essential features. Under these circumstances, I have been compelled to seek another channel for its publication.

It is, of course, always desirable in scientific matters to avoid personal controversy, but there are occasions in which personal explanations become necessary. The matter at issue between Mr. Durham and myself is whether a certain operation should be "earlier, more boldly, and more readily resorted to," as recommended by Mr. Durham, or whether it should be reserved for extreme cases, as advised by myself. The operation having been performed much more frequently abroad than in England, it becomes most important that the results of the operation in other countries should be accurately ascertained. In making my reports of these operations, I have been accused by Mr. Durham of numerous inaccuracies, and he has implied that I have "overstated the dangers and difficulties of the operation." On the other hand, I justify my reports, and point out the errors into which, I consider, Mr. Durham has fallen. It is thus that a scientific question has necessarily become a matter of personal controversy; and it will be seen that, whilst Mr. Durham has been allowed to make a personal attack on me, I have been prevented from replying to him at the Society where his attack was made, on the ground that my defence was personal and controversial.

The following paper is identical with that offered to the Royal Medical and Chirurgical Society; and the profession will now be in a position to form a judicial opinion, not only on the matter in dispute between Mr. Durham and myself, but also on the larger and more important question of the merits of thyrotomy for the removal of growths from the larynx.

In an article recently published in the Transactions of the Royal Medical and Chirurgical Society by Mr. Durham, "On Section of the Laryngeal Cartilages for the Removal of Morbid Growths," too favourable an estimate has, in my opinion, been taken of the operation of thyrotomy, whilst the correctness of my conclusions and the accuracy of my statements have been repeatedly impugned.

With a view of restoring the operation to its proper position, and of defending myself from the charges of inaccuracy, I venture to submit this paper to the consideration of the profession. The subject of thyrotomy, in so far as it refers to the removal of morbid growths, was first brought under the notice of the profession in this country by Sir Duncan Gibb, in a paper published in the British MEDICAL JourNAL, September 28th, 1865, which contained one case, under the joint care of himself and Mr. Holthouse, and a brief historical résumé of the subject.

In my essay on Grozuths in the Larynx, published in 1871, twenty pages were devoted to the treatment of morbid growths by "extralaryngeal" methods, and great pains were taken to form a just estimate of the value of thyrotomy. The work contained a table (p. 92), in which nearly all the published cases of thyrotomy were placed. The indications and contra.indications of the operation, together with its results as deduced from the table, were also placed before the profession, and it was laid down " as a cardinal law that an extra-laryngeal method ought never to be adopted (even where laryngoscopic treatment cannot be pursued), unless there be danger to life from suffocation or dysphagia."

Further, I expressed my belief "that the existence of dysphonia does not justify operations which, though easy to perform, may be regarded as capital," and that an "extra-laryngeal operation is not justifiable for the removal of a small growth in the larynx, unless that growth give rise to dangerous dyspnœa, and cannot be removed by a less serious method."

Six months after the publication of my work, Mr. Durham read his paper. In advocating the operation, Mr. Durham has not attempted to define the limits within which the operation should be performed. He neither confines it to cases in which there is dangerous dyspnœa or dysphagia, nor objects to its being done for the removal of small growths. He contents himself with enumerating the following propositions.

"First, that the dangers and difficulties attending it are neither so numerous nor so considerable as have been represented and commonly supposed; and

"Secondly, that the success hitherto achieved has been so marked and so indisputable, as to justify and encourage in any such case as may seem appropriate, an earlier, bolder, and more ready resort to this method than has hitherto prevailed."

In order to give the profession an opportunity of judging of the results of the operation, I have made out a fresh table of all the recorded cases of thyrotomy, and have arranged them in chronological order. In all instances where thyrotomy has been performed afresh, the original wound having completely healed up, the operation has been considered as a new case. All cases of cancer are printed in italics. [See Table.]

From an examination of this table my readers will be able to judge whether the dangers and difficulties of the operation have been overstated, and whether "an earlier, bolder, and more ready resort to this method" is to be encouraged. Instead of using the vague terms, "completely successful," "partially successful," "temporary benefit," and "negative," it will be better to consider the results of the operation, I. In relation to life; 2 . In relation to respiration; 3 . In relation to voice; 4 . In relation to recurrence.

In order to give an opportunity of reviewing, and, if necessary, of revising the facts on which my statements and statistics are based, in dealing with the results of the operation, I have furnished detailed lists of the cases considered under each heading.

With regard to the mortality after the operation, it appears that out of forty-eight cases two terminated fatally within a few days; two died at the end of a few weeks, and five succumbed at periods varying from six months to two years. In five of these fatal cases the disease was considered malignant.

The following is a list of the fatal cases, those of a carcinomatous character being printed in italics.

No. IC. Debrou, in 7 days, from metastatic abscesses.

No. 33. Schrötter, in I I days, from erysipelas and gangrene.

No. I. Brauers, in a few weeks, from hectic.

No. 8. Böckel, in 8 to 12 weeks, cause not stated.

No. 43. Mackenzie and Wordsworth, in 7 months, from exhaustion.

No. 46. Mackenzie and Thornton, in 7 months, from dysphagia.

No. I I. Gibb and Holthouse, in I year, from exhaustion.

No. 4. Gurdon Buck, in I 5 months, from suffocation.

No. 5. Rauchfuss, in 2 years, from perforation of cesophagus.

Two other patients (Ehrmann, No. 2, and Sands, No. 7) died from disease independent of the larynx, viz., one from typhus, the other from cancer of the kidneys and suprarenal capsules. Though both these patients remained dysphonic, and in one recurrence took place within seven months of the operation, I have entered their cases as recoveries in relation to life.

Omitting all those cases which survived the operation more than a few weeks, there remain four in which death may be attributed directly to the operation or its effects-a mortality of 8.33 per cent. Mr. Dur- 
TABLE SHOWING RESULTS OF ALL CASES OF THYROTOMY.

[All those cases printed in Italics are supposed to have been of a malignant or quasi-malignant character.]

\begin{tabular}{|c|c|c|c|c|c|c|c|c|c|c|c|}
\hline \multirow[b]{2}{*}{ No. } & \multirow[b]{2}{*}{ Date. } & \multirow[b]{2}{*}{ Operator. } & \multicolumn{3}{|c|}{ Before Operation. } & \multicolumn{6}{|c|}{ AFter Operation. } \\
\hline & & & Age. & Sex. & Symptoms. & & rtality & & Respiration. & Voice. & $\begin{array}{c}\text { Recurrence, or } \\
\text { Incomplete Removal. }\end{array}$ \\
\hline I & 1833 & $\begin{array}{l}\text { Brauers (Fournal de Gräfe } \\
\text { et Walther, } 1834 \text {, vol. xxi, } \\
\text { p. } 534) \text {. }\end{array}$ & 40 & M. & Dyspnœa & & Death. & & $\cdots \quad \cdots \quad \cdots$ & Aphonic & . \\
\hline 2 & \844 & $\begin{array}{l}\text { Ehrmann (Histoire des Po- } \\
\text { lypes du Larynx, } 1850 \text { ). }\end{array}$ & 33 & F. & $\begin{array}{l}\text { Dyspnœa and } \\
\text { aphonia }\end{array}$ & .. & $\cdot \cdot$ & $\cdot \cdot$ & Normal & Aphonic & In 7 months. \\
\hline 3 & $\begin{array}{l}\text { May } \\
1851\end{array}$ & $\begin{array}{l}\text { Gurdon Buck (New York } \\
\text { Med. Fournal, May r865). }\end{array}$ & $5 I$ & $F$ & $\begin{array}{l}\text { Dyspnoca and } \\
\text { aphonia }\end{array}$ & & .. & & $\begin{array}{c}\text { Canula always } \\
\text { worn }\end{array}$ & $\begin{array}{c}\text { Aphonic (" a whisper- } \\
\text { ing voice") }\end{array}$ & $\begin{array}{l}\text { Whole growth not } \\
\text { removed. }\end{array}$ \\
\hline 4 & $\begin{array}{l}\text { Sept. } \\
\text { r857 }\end{array}$ & $\begin{array}{c}\text { Gurdon Buck (New York } \\
\text { Med. Fournal, May 1865). }\end{array}$ & $5 I$ & $F$. & $\begin{array}{l}\text { Dyspnoea and } \\
\text { aphonia }\end{array}$ & $\begin{array}{l}\text { Death } i \\
\text { from for } \\
\text { from d }\end{array}$ & $\begin{array}{l}n \text { I5 } \\
\text { st oppe } \\
\text { isplace }\end{array}$ & $\begin{array}{l}\text { months } \\
\text { ration. } \\
\text { ement }\end{array}$ & $\begin{array}{c}\text { Canula zuorn till } \\
\text { death }\end{array}$ & Aphonic & $\begin{array}{ll}. & \end{array}$ \\
\hline 5 & 1861 & $\begin{array}{l}\text { Rauchfuss (St. Petersburg } \\
\text { Medizin. Zeitsch., I862, } \\
\text { vol. v, p. 44). }\end{array}$ & Adult & $\mathrm{F}$. & $\begin{array}{c}\text { Dyspnœa ; probably } \\
\text { aphonia }\end{array}$ & $\begin{array}{l}\text { Death in } \\
\text { perforati } \\
\text { into } \propto\end{array}$ & $\begin{array}{l}\text { canul } \\
2 \text { yea } \\
\text { on of } t \\
\text { esopha }\end{array}$ & $\begin{array}{l}\text { s from } \\
\text { achea } \\
\text { zus }\end{array}$ & $\begin{array}{l}\text { Canula always } \\
\text { worn }\end{array}$ & $\begin{array}{l}\text { Aphonic (right vocal } \\
\text { cord removed in } \\
\text { operation) }\end{array}$ & $\begin{array}{l}\text { Incomplete removal : } \\
\text { "soon sprang up } \\
\text { afresh". }\end{array}$ \\
\hline 6 & 1861 & $\begin{array}{l}\text { Gurdon Buck (New York } \\
\text { Med. Fournal, May 1865). }\end{array}$ & 25 & M. & Not stated & .. & .. & .. & $\begin{array}{l}\text { Canula always } \\
\text { worn }\end{array}$ & Aphonic (probably) & $\begin{array}{l}\text { Not stated, but a } \\
\text { "marked narrowing }\end{array}$ \\
\hline 7 & $r 863$ & $\begin{array}{l}\text { Sands (Newe York Medical } \\
\text { Fournal, May } 1855 \text { ). }\end{array}$ & 30 & $F$ & $\begin{array}{c}\text { Slight dyspnoxa and } \\
\text { dysphonia }\end{array}$ & $\cdots$ & . & & Normal & $\begin{array}{l}\text { Modified ("Resonant, } \\
\text { but not normal") }\end{array}$ & $\begin{array}{c}\text { of the rima resulted. } \\
. .\end{array}$ \\
\hline 8 & $\tau 863$ & $\begin{array}{l}\text { Böckel (Extrait de la Thèse } \\
\text { de Sivebel,Strasbourg, } 1866) \text {. }\end{array}$ & 24 & F. & $\begin{array}{c}\text { Dyspnca and } \\
\text { dysphonia }\end{array}$ & In 3 & mont & & Normal & Aphonic & $\begin{array}{l}\text { Recurrence of small } \\
\text { rosy projections in } \\
7 \text { weeks. }\end{array}$ \\
\hline 9 & 1863 & $\begin{array}{l}\text { Busch (Beobachtungen zur } \\
\text { inuern Klinil, Bonn, 1864, } \\
\text { p. xo8). }\end{array}$ & 43 & M. & $\begin{array}{l}\text { Dyspncea and } \\
\text { dysphonia }\end{array}$ & $\cdots$ & . & $\cdot \cdot$ & $\begin{array}{l}\text { Canula always } \\
\text { worn }\end{array}$ & Dysphonic & $\begin{array}{ll}7 & \text { weeks. } \\
. & .\end{array}$ \\
\hline xo & 1864 & $\begin{array}{l}\text { Debrou (Gazette des Hôpi- } \\
\left.\text { taux, May } 2, \mathrm{1} 86_{3}\right) \text {. }\end{array}$ & $5 \mathrm{I}$ & M. & $\begin{array}{l}\text { Dyspnoea and } \\
\text { dysphagia }\end{array}$ & Death & in $7 c$ & lays & $\begin{array}{lll}\cdots & \cdots & \cdots\end{array}$ & $\begin{array}{lll}\cdot & \cdots & \end{array}$ & ・. \\
\hline II & 1864 & $\begin{array}{l}\text { Gibb and Holthouse (Brit. } \\
\text { Med. Fournal, Sept. 28th, } \\
\text { I865). }\end{array}$ & 29 & $F$ & $\begin{array}{l}\text { Dyspnxa and } \\
\text { dysphonia }\end{array}$ & Death & in one & year & $\begin{array}{c}\text { Canula always } \\
\text { zoor } n\end{array}$ & $\begin{array}{l}\text { Clcar for four months; } \\
\text { afterwards aphonic. } \\
\text { Constant dysphagia }\end{array}$ & In 4 months. \\
\hline 12 & $186_{4}$ & $\begin{array}{l}\text { Lewin and Ulrich (Deutsche } \\
\text { Klinik, I865, No. 52, p. } \\
5 \text { 10). }\end{array}$ & I6 & $\mathrm{F}$. & $\begin{array}{l}\text { Dyspnoca and } \\
\text { aphonia }\end{array}$ & .. & .. & $\cdots$ & Normal & $\begin{array}{c}\text { Modified (bass voice } 22 \\
\text { days after; no further } \\
\text { history) }\end{array}$ & . \\
\hline$x_{3}$ & 1864 & $\begin{array}{l}\text { Gilewski (Wiencr, Medizin. } \\
\left.\text { Woch., June } 28,{ }^{2} 65, \mathrm{p} . \mathbf{1}^{2}\right)\end{array}$ & 16 & F. & $\begin{array}{l}\text { Dyspnoca and } \\
\text { dysphonia }\end{array}$ & .. & .. & . & Normal & Dysphonic & .. \\
\hline 14 & $\begin{array}{l}\text { Feb. } \\
\text { I } 865\end{array}$ & $\begin{array}{l}\text { Gouley }(\text { Neru York Medical } \\
\text { Four., Sept. 1867, p. 473). }\end{array}$ & 6 & F. & $\begin{array}{l}\text { Dyspnœa and } \\
\text { aphonia }\end{array}$ & . & . & . & $\begin{array}{l}\text { Normal for six } \\
\text { months }\end{array}$ & Aphonic("whisper") & In 6 months. \\
\hline 15 & 1865 & $\begin{array}{l}\text { Balassa (Wiener Medizin. } \\
\text { Wochenschr., Nov, r868). }\end{array}$ & 44 & F. & $\begin{array}{l}\text { Dyspnœea, aphonia, } \\
\text { and dysphagia. }\end{array}$ & $\cdots$ & . & $\cdot \cdot$ & Normal & Normal & Recurrence. \\
\hline$x 6$ & 1865 & $\begin{array}{l}\text { Kürbele (Gazette des Hôpi- } \\
\left.\text { taux, June }{ }_{3}, 1865\right) \text {. }\end{array}$ & 57 & M. & $\begin{array}{l}\text { Dyspnoca, aphonia, } \\
\text { and dysphagia }\end{array}$ & .. & . & .. & $\begin{array}{l}\text { Canula always } \\
\text { worn }\end{array}$ & Aphonic & $\cdots \quad \cdots$ \\
\hline I7 & $\begin{array}{l}\text { Nov. } \\
\text { r } 865\end{array}$ & $\begin{array}{l}\text { Gouley (New York Medical } \\
\text { Four., Sept. 1867, p. 473). }\end{array}$ & 7 & F.' & $\begin{array}{l}\text { Dyspnœea and } \\
\text { aphonia }\end{array}$ & . & .. & .. & Normal & $\begin{array}{l}\text { Aphonic ("a loud very } \\
\text { distinct whisper") } \\
\text { July } 867\end{array}$ & $\begin{array}{l}\text { Some irregularity of } \\
\text { vocal cord, but no }\end{array}$ \\
\hline 18 & 1866 & $\begin{array}{l}\text { Balassa( Wiener Med. Woch., } \\
\text { ı } 868, \text { No. 93). }\end{array}$ & 32 & M. & $\begin{array}{l}\text { Dyspnoca and } \\
\text { dysphonia }\end{array}$ & $\cdots$ & .. & .. & Normal & $\begin{array}{l}\text { July } 1867 \\
\text { Normal }\end{array}$ & $\begin{array}{l}\text { recurrence. } \\
\text { Cure complete in } 8 \\
\text { days; no history }\end{array}$ \\
\hline Ig & 1866 & $\begin{array}{l}\text { Voss (Medico-Chir. Trans., } \\
\text { vol. lv, p. 87). }\end{array}$ & $4 \frac{1}{2}$ & M. & Dysphonia & $\cdots$ & . & .. & Normal & Not stated & $\begin{array}{l}\text { after that date. } \\
\text { Recurrence in } 6 \\
\text { months. }\end{array}$ \\
\hline 20 & 1866 & $\begin{array}{l}\text { Durham (Guy's Hospital K' } \\
\text { ports, } 1866) \text {. }\end{array}$ & 13 & $F$ & $\begin{array}{l}\text { Dyspnoca and } \\
\text { aphonia }\end{array}$ & . & .. & .. & Normal & Normal & $\begin{array}{ll}. & \end{array}$ \\
\hline $2 I$ & $x 867$ & $\begin{array}{l}\text { Balassa(Wiener Med. Woch., } \\
\text { Nov. I I, 1868). }\end{array}$ & 19 & F. & $\begin{array}{l}\text { Dyspnœea and } \\
\text { dysphonia }\end{array}$ & . & .. & *. & Normal & Normal & In 3 months. \\
\hline 22 & 1867 & $\begin{array}{l}\text { Voss (Medico-Chir. Trans., } \\
\text { vol. Iv, } \mathrm{p}, 87 \text { ). }\end{array}$ & 4 & M. & Dysphonia & . & . & -. & Normal & Not stated & .. \\
\hline 23 & 1867 & $\begin{array}{l}\text { Long (Liverpool Hospital } \\
\text { Reports, 1867). }\end{array}$ & 8 & M. & Dyspnœa & .. & .. & .. & Normal & $\begin{array}{l}\text { Dysphonic ("somewhat } \\
\text { hoarse, especially when } \\
\text { he gets a little cold") }\end{array}$ & . \\
\hline 24 & 1867 & $\begin{array}{l}\text { Balassa (Wiener Medezin. } \\
\text { Wochenschr. No. } 92,1868 \text { ). }\end{array}$ & $2 \mathrm{I}$ & F. & $\begin{array}{l}\text { Dyspnœa and } \\
\text { aphonia }\end{array}$ & .. & $\cdots$ & 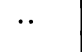 & $\begin{array}{l}\text { Normal for a few } \\
\text { months }\end{array}$ & Dysphonic & $\begin{array}{l}\text { Recurrence soon } \\
\text { after operation. }\end{array}$ \\
\hline 25 & 1867 & $\begin{array}{l}\text { Holmes (Surg. Treatment } \\
\text { of Children's Diseases, and } \\
\text { edition, p. } 3 \mathrm{rI} \text { ). }\end{array}$ & 9 & F. & $\begin{array}{l}\text { Dyspnoca and } \\
\text { aphonia }\end{array}$ & $\cdots$ & ・. & . & $\begin{array}{l}\text { Canula always } \\
\text { worn }\end{array}$ & Aphonic & Not stated. \\
\hline 26 & 1867 & $\begin{array}{l}\text { Cutter (Boston Medical and } \\
\text { Surg. Four., Feb. I86g). }\end{array}$ & 53 & M. & $\begin{array}{l}\text { Dyspnoea and } \\
\text { dysphonia }\end{array}$ & . & $\cdots$ & $\cdots$ & Normal & $\begin{array}{l}\text { Modified ("coarse } \\
\text { and clear") }\end{array}$ & $\begin{array}{l}\text { Soon after; date } \\
\text { not stated. }\end{array}$ \\
\hline 27 & х868 & $\begin{array}{l}\text { Balassa (Wiener Med. Woch., } \\
\text { No. } 92, \text { 1868). }\end{array}$ & 22 & F. & $\begin{array}{l}\text { Dyspnœea and } \\
\text { aphonia }\end{array}$ & .. & .. & .. & Normal & Normal & . $\quad \cdots$ \\
\hline 28 & I868 & $\begin{array}{l}\text { Mackenzie and Couper (Es- } \\
\text { say on Groweths in the } \\
\text { Larynx. Case } 64) \text {. }\end{array}$ & 65 & $\mathrm{~F}$. & $\begin{array}{l}\text { Dyspnoca and } \\
\text { aphonia }\end{array}$ & . & $\cdots$ & $\cdots$ & $\begin{array}{l}\text { Normal for } 2 \frac{1}{2} \text { years } \\
\text { (afterwards } \\
\text { dyspnœa) }\end{array}$ & $\begin{array}{l}\text { Normal for } 2 \frac{1}{2} \text { years, } \\
\text { (afterwards dysphonic) }\end{array}$ & In $2 \frac{1}{2}$ years. \\
\hline 29 & 1868 & $\begin{array}{l}\text { Mackenzie and Evans (Essay } \\
\text { on Growths in the Larynx. } \\
\text { Case } 69 \text { ). }\end{array}$ & 12 & F. & $\begin{array}{l}\text { Dyspnoa and } \\
\text { aphonia }\end{array}$ & $\cdots$ & . & ・. & Normal & Aphonic & . \\
\hline 30 & 1868 & $\begin{array}{l}\text { Navratil(BerlinKlin. Woch., } \\
\text { I } 868 \text {, No. 49, p. 50r). }\end{array}$ & Adult & M. & Not stated & & & & Normal & $\begin{array}{l}\text { Dysphonic ("voice } \\
\text { harsh and hollow") }\end{array}$ &.$\cdot$ \\
\hline $3 \mathrm{I}$ & 1868 & Navratil (Ibid., p. 5or). & 20 & F. & $\begin{array}{l}\text { Dyspnoca and } \\
\text { aphonia }\end{array}$ & . & $\cdots$ & & $\begin{array}{c}\text { Canula always } \\
\text { worn }\end{array}$ & & $\begin{array}{l}\text { Growth not extir- } \\
\text { pated. }\end{array}$ \\
\hline $\begin{array}{l}32 \\
33\end{array}$ & $\begin{array}{l}1868 \\
\mathbf{1 8 6 9}\end{array}$ & $\begin{array}{l}\text { Navratil (16id., p. 502). } \\
\text { Schrötter (Medezin. Fahr- } \\
\text { buch, Wien, r869, vol. xvii, } \\
\text { 2nd Heft, p. 8I). }\end{array}$ & $\begin{array}{l}30 \\
63\end{array}$ & $\begin{array}{l}M . \\
M\end{array}$ & $\begin{array}{l}\text { Not stated } \\
\text { Dyspnaca and } \\
\text { dysphonia }\end{array}$ & $\begin{array}{l}\text { Death in } \\
\text { erysif } \\
\text { ga }\end{array}$ & $\begin{array}{l}\ddot{\text { II da }} \\
\text { pelas } \\
\text { ngren }\end{array}$ & $\begin{array}{l}\text { s̈rom } \\
\text { nd }\end{array}$ & $\begin{array}{l}\text { Normal } \\
\text { Canula always } \\
\text { zoorn }\end{array}$ & $\begin{array}{l}\text { Dysphonic } \\
. . \\
\cdots\end{array}$ & $\begin{array}{l}\text { Growth not extir- } \\
\text { pated. }\end{array}$ \\
\hline 34 & 1869 & $\begin{array}{l}\text { Mackenzie and Wordsworth } \\
\text { (Essay on Grozuths in the } \\
\text { Larynx. Case } 87) \text {. }\end{array}$ & 47 & $M$ & $\begin{array}{l}\text { Dyspnax, dysphonia, } \\
\text { and dysphagia }\end{array}$ & Death & in $7 \mathrm{~m}$ & onths & $\begin{array}{c}\text { Canula always } \\
\text { zuorn }\end{array}$ & Aphonic & In 2 months. \\
\hline 35 & 1869 & $\begin{array}{l}\text { Cohen (New York Medical } \\
\text { Record, August } 16,1869) \text {. }\end{array}$ & Adult & M. & Dysphonia & $\cdots$ & ・. & . & Normal & Dysphonic & Recurrence. \\
\hline 36 & ז869 & $\begin{array}{l}\text { Krishaber (Gazette des Hôpi- } \\
\text { taux, 1869, No. ro3). }\end{array}$ & $3^{8}$ & M. & $\begin{array}{l}\text { Dyspnoca and } \\
\text { dysphonia }\end{array}$ & .. & .. & .. & Normal & Normal & .. \\
\hline 37 & 1870 & $\begin{array}{c}\text { Gurdon Buck (Trans. of Nenv } \\
\text { Yk. Acad.of Med.,v.iii,pt. Io) }\end{array}$ & $3^{8}$ & M. & $\begin{array}{l}\text { Dyspnoca and } \\
\text { dysphonia }\end{array}$ & . & . & $\cdots$ & Normal & $\begin{array}{l}\text { Dysphonic (rt. v. c. and } \\
\text { aryt. cart. removed) }\end{array}$ & *. \\
\hline $3^{8}$ & 1870 & $\begin{array}{l}\text { Denucé (Bordeaux Médi- } \\
\left.\text { cale, Feruary } 1_{5}, 1_{72}\right) \text {. }\end{array}$ & 54 & F. & $\begin{array}{l}\text { Dyspnoca and } \\
\text { dysphonia }\end{array}$ & . & . & *. & Normal & Normal & . \\
\hline
\end{tabular}


TABLe of Throtomi, continue?!

\begin{tabular}{|c|c|c|c|c|c|c|c|c|c|c|c|}
\hline \multirow{3}{*}{ No. } & \multirow{3}{*}{ Date. } & \multirow[b]{2}{*}{ Operator. } & \multicolumn{3}{|c|}{ BEFORE OPERATION. } & \multicolumn{6}{|c|}{ After Operation. } \\
\hline & & & Age. & Sex. & Symptoms. & \multicolumn{3}{|c|}{ Mortality. } & \multirow{2}{*}{ Respiration. } & \multirow{4}{*}{$\begin{array}{l}\text { Voice. } \\
\text { Modified ("'parents } \\
\text { quite satisfied with } \\
\text { condition of voice") } \\
\text { Modified ("clear, but } \\
\text { rather feeble") } \\
\text { Normal }\end{array}$} & $\begin{array}{l}\text { Recurrence, or } \\
\text { Incomplete Removal. }\end{array}$ \\
\hline & & $\begin{array}{l}\text { Durham (.Medico-Chiru'g. } \\
\text { Truns., vol. lv, p. 2o). }\end{array}$ & 7 & M. & $\begin{array}{l}\text { Dyspnoea and } \\
\text { aphonia }\end{array}$ & $\cdots$ & $\cdots$ & .. & & & .. \\
\hline 40 & 1870 & $\begin{array}{l}\text { Durham (Mcdico-Chirurg. } \\
\text { Trans., vol. lv, p. 22). }\end{array}$ & 8 & F. & $\begin{array}{l}\text { Dyspnoea and } \\
\text { aphonia }\end{array}$ & $\cdots$ & $\cdots$ & $\bullet$ & Normal & & $\cdots$ \\
\hline 41 & 1870 & $\begin{array}{l}\text { Bryant (Medico-Chirurgical } \\
\text { Trans., vol. lv, p. 24.) }\end{array}$ & 3 & M. & $\begin{array}{l}\text { Dyspncea and } \\
\text { aphonia }\end{array}$ & $\cdots$ & $\cdots$ & $\bullet$ & Normal & & $\cdots$ \\
\hline 42 & 1871 & $\begin{array}{l}\text { Langenbeck (British Medi- } \\
\text { calfournal, Nov. 4, 1872). }\end{array}$ & 28 & M. & Dyspnœa & .. & .. & $\cdots$ & Normal & $\begin{array}{c}\text { Normal (voice not af- } \\
\text { fected prior tooperation) }\end{array}$ & .. \\
\hline 43 & $\begin{array}{l}\text { Aug. } \\
1871\end{array}$ & Davies-Colley (Medico-Chir. & 4 & M. & $\begin{array}{l}\text { Dyspnoea and } \\
\text { aphonia }\end{array}$ & $\cdots$ & $\cdots$ & *. & $\begin{array}{c}\text { Canula always } \\
\text { worn }\end{array}$ & $\begin{array}{l}\text { Aphonic ("a husky, } \\
\text { loud, whispering voice") }\end{array}$ & $\begin{array}{l}\text { Recurrence in a few } \\
\text { months. }\end{array}$ \\
\hline 44 & 1871 & $\begin{array}{l}\text { Ogle and Lee, (Med.-Chir. } \\
\text { Trans., vol. lv, p. 28). }\end{array}$ & 5 & M. & Aphonia & -. & $\cdots$ & .. & Normal & Normal & $\begin{array}{ll}\cdots & \cdots\end{array}$ \\
\hline 45 & 1872 & $\begin{array}{l}\text { Mackenzie and Thornton } \\
\text { (Read at Clinical Society, } \\
\text { February 14, 1872). }\end{array}$ & 24 & M. & $\begin{array}{l}\text { Dyspnoca and } \\
\text { aphonia }\end{array}$ & & mon & & $\begin{array}{c}\text { Canula alzuays } \\
\text { worn }\end{array}$ & Aphonic & In 4 months. \\
\hline $4^{6}$ & $\underset{1872}{\operatorname{March}}$ & $\begin{array}{l}\text { Davies-Colley (Medico-Chir. } \\
\text { Trans., vol. lv, p. 27). }\end{array}$ & 5 & M. & $\begin{array}{l}\text { Dyspnoea and } \\
\text { aphonia }\end{array}$ & $\cdots$ & 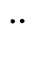 & - & Dyspnœa & $\begin{array}{l}\text { Dysphonia (" voice } \\
\text { audible, but by no } \\
\text { means natural") }\end{array}$ & $\begin{array}{l}\text { Recurrence shortly } \\
\text { after. }\end{array}$ \\
\hline 47 & 1872 & $\begin{array}{l}\text { Semple and Thornton (Real } \\
\text { at Clin.Soc., Feb.14, 1872). }\end{array}$ & $2 \frac{1}{2}$ & M. & $\begin{array}{l}\text { Dyspnœea and } \\
\text { aphonia }\end{array}$ & $\cdots$ & $\cdots$ & $\because$ & $\begin{array}{l}\text { Normal for } 3 \text { months; } \\
\text { canula reinserted } \\
\text { in } 6 \text { months }\end{array}$ & Aphonic & $\begin{array}{l}\text { Recurrence in } 3 \\
\text { months ; readmitted } \\
\text { January } 1,1873 \text {. }\end{array}$ \\
\hline $4^{8}$ & $\begin{array}{l}\text { Sept. } \\
1872\end{array}$ & $\begin{array}{l}\text { Davies-Colley (British Me- } \\
\text { dical fonrnal, Sept. 1872). }\end{array}$ & $5 \frac{1}{2}$ & M. & $\begin{array}{l}\text { Dyspnœea and } \\
\text { aphonia }\end{array}$ & -・ & $\cdots$ & $\because$ & $\begin{array}{c}\underset{\text { Canula always }}{\text { worn }} \\
\text {. }\end{array}$ & No report & No report. \\
\hline
\end{tabular}

ham only admits two deaths as resulting "more or less directly from the operation ;" and, as far as I can understand, they are those of Schrötter and Debrou. To the former case I shall presently refer. In Debrou's case, after division of the thyroid cartilage, a tracheotomy tube was inserted, and, in accordance with the usual practice, left in sitı. The patient died seven days after the operation from meta. static abscesses of the lungs. Debrou attributed the death to the use of the tracheal canula, and this ingenious explanation of the fatal issue has been considered reasonable by Mr. Durham. No further comment is required on this case.

The fatal cases which Mr. Durham excludes are those of Brauers and Böckel. The facts concerning these cases are as follows. Brauers' case was first published in the Fournal de Gräfe et Walther in 1834, and in 1850 was included by Ehrmann in his classical Histoire des Polypes du Larynx (vol. xxi, p. 534). The patient's larynx was opened by Brauers on several occasions, and the growths treated with acid nitrate of mercury, actual cautery, etc. Nevertheless the growths returned; and, to use the exact words of the original report, "as the result of successive irritations produced by repeated cauterisations, the larynx passed into a state of scirrhous induration, hectic fever supervened, which must necessarily lead to death, ${ }^{*}$ although this had not yet happened at the epoch when the physician, the reporter of this case, gave us his account at Bonn." The exact expression in French is "devait amener necessairement la mort," that is, "must necessarily lead to death ;" Mr. Durham has greatly qualified the expression by translating it, "which seemed almost certain to prove fatal." When it is borne in mind that Ehrmann was justly considered the greatest authority on diseases of the larynx, and that he was strongly in favour of thryrotomy, there cannot be the least doubt that had Brauers' patient survived, Ehrmann would have obtained knowledge of it. I have considered this as a fatal case. Mr. Durham, however, on the authority of Krishaber, stated that " the patient lived more than twenty years, and died of a disease quite foreign to the larynx."

As Dr. Krishaber produced no evidence of the survival of the patient, I wrote to him, and received the following reply.

"I read the statement in a Vienna medical journal in 1866 , and signed, I believe, by Gilewski, that Brauers' patient survived the operation twenty years." $\dagger$

After careful examination, I have not been able to discover any paper by Gilewski in a Vienna medical journal in 1866, but he wrote an article in the Wiener Medizinische Wochenschrift, June 28th and July Ist, 1865, and therein he stated that his own case and those of Ehrmann and Rauchfuss were the only three in which the operation had been performed. He was, therefore, at that date, altogether ignorant of the existence of Brauers' case. It would have been strange, indeed, if Dr. Gilewski, practising in the south of Poland, could have obtained evidence concerning the recovery of a patient who was supposed to have died thirty-three years previcusly in Belgium. It will

* The italics are not in the original.

+ On receipt of Dr. Krishaber's letter, I wrote to Dr. Gilewski, asking if he had ever made such a statement, and, if so, on what authority; but, in conseq uence of Dr. Gilewski's death, the letter was returned to me through the post-office. be seen, therefore, that there is no evidence whatever of the survival of Brauers' patient, but that all the evidence points to his death.

In Böckel's case (No. 8) the patient left the hospital after the operation, and died a few weeks afterwards, the exact cause of her death not having been ascertained. Notwithstanding that the patient was apho. nic, that she had suffered from perichondritis as a result of the operation, that recurrence had already taken place- "small rosy projections" having been seen on laryngoscopic examination and cauterised-not. withstanding that, even when she left the hospital, she had a laryngeal fistula, and that she died so suddenly five or six weeks later, that the medical practitioner could not reach her before death, Mr. Durham quotes from the reporter that "it is probable that this unfortunate woman* succumbed to some intercurrent affection of her lungs, which she contracted in the rude climate of her valley," and himself adds, that "there is no reason for supposing that the death was in any way due to the operation ;" but, I venture to think, it will be generally considered that the operation of thyrotomy or its results had a more immediate relation to the death of this "unfortunate woman" than "the rude climate of her valley."

Before dismissing this review of the fatal cases, I must call attention to Ehrmann's own case, my treatment of which does not appear suffrciently clear to Mr. Durham.

He states that "it is not quite clear whether Mackenzie reckons this as a fatal case of thyrotomy or not." Now, as there were ten fatal cases in my table, and I only reckoned nine deaths as resulting from thyrotomy or from disease of the larynx, it is evident that I did not include Ehrmann's as a fatal case. But, in addition to this, I expressly stated that "the case was justly considered one of recovery, and that death took place from typhus" (Op. cit., note to p. 97). It would be difficult, in my opinion, to use language much more clear.

Dr. Sands's case (No. 7), which I have placed amongst those of malignant character (although it is extremely doubtful whether the growth removed from the larynx was really cancerous), was certainly a recovery as far as mortality is concerned. The patient died twenty-two months after the operation, having, however, always remained aphonic.

The actual mortality does not, however, give an adequate idea of the danger of the operation, for among these cases there were many " hair-breadth 'scapes." Thus, in Dr. Cutter's case (No. 27), (Boston Medical and Surgical fournal, Feb. 18th, 1869), the patient was almost suffocated during the operation; and, to use Dr. Cutter's own words, the return to complete sensibility was retarded by the accumulation of blood and mucus in the mouth, which ran down the trachea, and out of the artificial opening; it was also accompanied by profuse sweating and some flagging of the pulse." After the patient was put to bed "vomiting ensued, and a large amount of blood, mingled with mucus, was evacuated."

In one of Navratil's cases (No. 30), (Berlin Klin. Wochenschrift,

"The "unfortunate woman", on leaving the hospital, returned to the "rude climate of her native valley", Munster, in the Haut Rhin. After a shorc time, Dr. Dietz of her native valley", Munster, in the Haut Rhin. After a short time, Dr. Diet
was summoned to attend her; but "death took place before the doctor could arrive at the patient's bed-side."-Mémoires de la Société de Chirurgie de Par is, tome page $56 r$. 
Dec. 7 th, 1868, p. 502), the hæmorrhage was alarming, and the patient nearly died under the operation, from the quantity of blood which passed down the trachea. In another of Navratil's cases (No. 32), (Ilid.), the patient suffered from high fever after the operation, and expectorated a quantity of blood and pus ; œdema took place round the wound, and the patient was in a very critical state.

In Schrötter's case (Medizin. Fahrbuch, Wien, 1869, vol. xvii, and Heft, p. 8I), the operator observed that, after dividing the thyroid cartilage, "holding open the borders of the wound with blunt hooks gave rise to such paroxysms of coughing and caused so much fresh hæmorrhage, that the examination could only be carried out for a short time;" and, further, "that the sputa consisted of pure blood even well into the night, and on the following day the expectoration was still coloured." By a clerical error in my thyrotomy table, it was stated that this patient died in seven hours instead of eleven days, as it should have been. I regret this error extremely; because, though original y occurring in an abbreviated tabular statement, it was subsequently accepted as a fact, and repeated in the text.

It is certainly remarkable that Mr. Durham, who has devoted nearly a page to the exposure of this clerical error of mine, and who has given up another page and a half to the description of Schrötter's case, besides referring to it on another separate occasion (Op. cit., p. 29), does not once mention the prolonged and dangerous hæmorrhage which occurred, and which must have greatly prejudiced the issue of the case, even if it did not, as I believe it did, directly lead to death.

Mr. Timothy Holmes remarks with regard to his case (Surgical Treatment of Childrin's Discascs, 2nd edit., p. 3II), "that the parts over the larynx were found to be peculiarly vascular." After the hæmorrhage caused by the preliminary incision had been stopped, the thyroid cartilage was divided. "The bleeding that followed was very considerable."

Again, the reporter of Mr. Davies-Colley's third operation (BRITISH Medical Journal, Sept. 28, 1872) remarks, "that the boy at one time ceased to breathe, blood having apparently run down the trachea into the bronchial tubes, and the chloroform acting powerfully on the lungs. But after artificial respiration had been carried on for several minutes the little patient recovered."

Mr. Durham even tries to qualify the only deaths he has at all admitted in the following words : "In each of these [cases] the fatal result was brought about in a manner by no means special to the operation, but, alas! of far too common occurrence in general surgical experience."

Upon this I have only to remark that when blood-poisoning ceases to follow operations, no doubt many surgical procedures will be adopted which are not at present in vogue; but that until that time arrives, septicemia remains one of the contingent risks of all operations. On the other hand, the life of the patient is not imperilled by this danger when laryngoscopic treatment is adopted.

In referring to my observations on the mortality of thyrotomy, $\mathrm{Mr}$. Durham has produced a very erroneous impression as regards my treatment of the subject. He has made it appear as if, whilst comparing the mortality of laryngoscopic treatment with that of thyrotomy, I have concealed the circumstance that the laryngoscopic cases in my essay were all benign, and that the thyrotomy table included some cases of cancer. By inference he leads his readers to suppose that $I$ have made an unfair comparison between the two sets of cases. Mr. Durham remarks as follows:- "Considering the prospect of the operation in relation to the preservation of life, Dr. Mackenzie says, in division of the laryngeal cartilages, there is always some immediate danger to life, and nine out of the twenty-eight cases on record terminated fatally." If Mr. Durham wished to do justice to my views, it is strange that he quoted an isolated passage, and did not add the remarks on the same page (94) viz., "In six of the nine fatal cases in the thyrotomy table the disease was cancerous (or semi-malignant)."

So far from attempting to conceal the inclusion of malignant cases, I called special attention to their admission, not only in the passage referred to, but also in almost the same words when spenking of recurrence*, where I not only gave prominence to the inclusion of cancer cases, but pointed out the pathological character of the growths as a cause of their great disposition to recurrence. I also called attention to the inclusion of cancer cases at the head and foot of my thyrotomy table. It is certainly remarkable that $\mathrm{Mr}$. Durham, who objects to my mode of dealing with the subject has himself adopted precisely the same plan, without any of the precautions which I have taken. In the fourth volume of Holmes's Systcm of Surgery, page 584, Mr. Durham has published a table showing " the general success of different methods of

* The following is the expression used by me:- "In six other cases, the patient died at the end of a few months; and in nearly all of these, recurrence had taken place. They were all, however, of malignant or semi-malignant character, and therefore the tendency to reproduction was no doubt very great."-Op. cit., p. 97 . operating." In this table "operation through the mouth," that is, laryngoscopic methods of treatment are compared with "operations after external incision ;" but Mr. Durham has not called attention to the inclusion of cancer cases amongst the operations after external incision. It will be seen, therefore, that whilst Mr. Durham finds fault with me for a mistake which I have not made, he has himself made the very mistake that he imputes to me.

[To be continucd. $]$

\section{ON THE TREATMENT OF SUPPOSED STRICTURE OF THE CERVIX UTERI.}

\section{By WILliam CUMMING, F.R.C.P.E., Edinburgh.}

TRUE stricture of the cervix uteri is rare ; not so rare, certainly, as is stricture of the urethra in the male who has not had gonorrhoa, but nearly so. Incision of the cervix, or hysterotomy, therefore, for the cure of stricture, should be one of the rarest operations in obstetric surgery. But of late years it has not been so ; and it may be a legitimate inquiry, what purpose it has served when the consequences seemed to sanction its use, which unquestionably they often do.

I. There may be, or rather there is often, a quasi-stricture at the internal os, when there is congestion, hypertrophy, or other disease of the lining membrane of the uterus. This condition is relieved tem. porarily-it may even be cured permanently-by the hæmorrhage resulting from the incision. In my own experience, the relief generally is only temporary.

2. The same condition of quasi-stricture exists when there is hypertrophy of the body of the uterus-in many instances induced by the efforts to expel the clotted menstrual and the accumulating leucorrhœal discharges. In such cases, the hæmorrhage, coupled with adequate general treatment, reduces the hypertrophy, prevents accumulation, and helps to restore the healthy state of the lining nuembrane, and so relieves the supposed stricture.

3. The preceding condition is almost invariably connected with enlargement and congestion of the ovaries, either as cause or as effect; and the same hæmorrhage reduces and relieves this.

But the operation, if effectually and thoroughly performed, is one of very considerable risk, especially when followed by the introduction of sponge- or tangle-tents. The interesting mechanical, or rather dynamical, experiments of Dr. Matthews Duncan prove with what force these bodies act ; and it is not difficult to estimate (if it were not sufficiently and disastrously confirmed by experience) the mischief that must in too many cases result from the laceration and irritation of the delicate textures cut into by the incision. How often this mischief has succeeded the operation, probably no one knows. Is, therefore, the operation altogether unjustifiable? I do not say so ; but, as a cure of this quasistricture, it should be almost the rarest operation in surgery. Is it justifiable as a cure or relief of the conditions I have alluded to above? I believe not, for three reasons: I. Because it does not, as a rule, either cure or more than temporarily relieve these morbid states; 2 . Recause it is always attended with danger; and that danger is increased the nearer the incision approaches to the internal os and the various plexuses adjoining, where the supposed need for the operation exists ; 3 . Because there is a much more simple, and, in my opinion and experience, less dangerous, treatment that may be employed with a larger probability of success. I cannot say utterly without risk, because every gynæcologist knows that, while there are some uteri to which almost anything may be done with impunity, there are others to which nothing can be done without startling, even fatal, results. I have known the gentle introduction of a moderate sized bougie lead to violent metritis, extending to the appendages, and not recovered from without permanent mischief; and I have also been cognisant of the same leading to acute suppuration of the ovary. But these results are so rare that, except as warnings to be both preparatory and cautious, they need scarcely be taken into account; and my present belief is, that if the gentle depletion I am disposed to advocate were employed before using the bougie, such complications would, probably, never occur.

Assuming, therefore, that true stricture is extremely rare, and that incision of the ora and cervix uteri, with subsequent dilatation by tents, is unnecessary, and may be, and often is, injurious, but that the hremorrhage resulting from it is beneficial, I have long practised, with fair success, scarification of the os uteri, followed by dilatation with graduated bougies. By scarification the amount of blood abstracted can be limited; as much or as little can be taken away as is thought necessary (which with leeches cannot be done) ; congestion of the lining membrane of the womb and of the ovaries is relieved and the pas- 